\title{
Certolizumab-Induced Uveitis: A Case Report and Review of the Literature
}

\author{
Elad Moisseiev Shiri Shulman \\ Department of Ophthalmology, Tel Aviv Medical Center, affiliated to the Sackler School of \\ Medicine, Tel Aviv University, Tel Aviv, Israel
}

\section{Key Words}

Certolizumab · Ocular sarcoidosis · Sarcoidosis · Uveitis · Tumor necrosis factor alpha

\begin{abstract}
We report the case of a 64-year-old woman with rheumatoid arthritis and bilateral visual deterioration. The patient had been treated with certolizumab, a new tumor necrosis factor alpha (TNF $\alpha$ ) antagonist, and her findings were consistent with bilateral uveitis, suggestive of sarcoidosis. Here, we review the literature on TNF $\alpha$ antagonist-induced sarcoidosis and report the first case of uveitis induced by certolizumab. Awareness of the possibility of this unique complication is important for both rheumatologists and ophthalmologists who treat patients with this new agent.

(C) 2014 S. Karger AG, Basel
\end{abstract}

\section{Introduction}

Tumor necrosis factor alpha (TNF $\alpha$ ) antagonists are an effective treatment for a variety of inflammatory and autoimmune diseases, and they are commonly administered to patients with rheumatoid arthritis (RA), ankylosing spondylitis and psoriatic arthritis [1]. However, in some cases, treatment with TNF $\alpha$ antagonists induces the onset of a new autoimmune disease. As these agents are being used for an expanding number of diseases and for longer periods, this phenomenon has also been increasingly noticed. These agents have been reported to induce lupus, vasculitis, interstitial lung disease, psoriasis, sarcoidosis, autoimmune hepatitis and myopathy $[1,2]$.

Sarcoidosis is characterized by noncaseating granulomas, and although it primarily affects the lungs and lymph nodes, any organ may be involved [3]. Induced sarcoidosis has been reported in association with infliximab, etanercept and adalimumab [1, 2, 4]. Certoli- 
Moisseiev et al.: Certolizumab-Induced Uveitis: A Case Report and Review of the Literature

zumab is a new TNF $\alpha$ antagonist which has been proven to be effective in the treatment of RA [5]. A review of the literature revealed no previous occurrence of sarcoidosis induced by this agent. We report the first case of certolizumab-induced uveitis, highly suggestive of ocular sarcoidosis, and review the literature relevant to the pathogenesis of this adverse effect.

\section{Case Report}

A 64-year-old woman was referred to our clinic due to bilateral reduced visual acuity (VA), mainly in her left eye. The patient's medical history was significant for seropositive RA, which had been well controlled for the previous 3 years under treatment with methotrexate and certolizumab (Cimzia). The patient had no previous ocular history.

The patient complained of a mild reduction in VA in both eyes during the previous year and noticed a significant reduction in her left eye (which occurred 1 week prior to her presentation). Initial examination revealed a VA of 20/32 in the right eye and 20/100 in the left eye. Anterior segment examination revealed no corneal keratic precipitates, mild $(+1)$ flare in the anterior chambers and posterior synechiae bilaterally (fig. 1a, b). Dilated fundus examination revealed vitreal cells and haze, which were present bilaterally, but more distinct in the left eye. Macular edema (fig. 1c, d) and peripheral retinal punched-out lesions were visible (fig. 1e). Intraocular pressure (IOP) was $21 \mathrm{~mm} \mathrm{Hg}$ in the right eye and $30 \mathrm{~mm}$ Hg in the left eye.

The integration of the clinical signs at presentation led to a working diagnosis of ocular sarcoidosis, possibly associated with anti-TNF $\alpha$ treatment. Workup included normal complete blood count, chemistry and C-reactive protein. Angiotensin-converting enzyme (ACE) levels were mildly elevated (61 U/l, normal range 10-51 U/l). Physical examinations and chest X-rays were normal, and so was the pulmonary function test. The patient was also examined by an expert pulmonologist who ruled out any lung disease.

She was treated with topical steroid drops as well as anti-glaucoma medication. By doing so, an improvement of the anterior uveitis and a normalization of the IOP were achieved. In coordination with the rheumatologist, the certolizumab was discontinued and the methotrexate dosage was increased. The topical steroidal treatment was tapered down and discontinued. Two months after the discontinuation of certolizumab, the uveitis had decreased and VA had improved to 20/32 in both eyes. Nevertheless, the macular edema persisted and the patient underwent periocular injections of $40 \mathrm{mg}$ triamcinolone acetonide (Triesence; Alcon Inc.) in both eyes, with a partial resolution of the macular edema (fig. 2).

One year after her initial presentation, the patient's RA is well controlled, and the change in systemic treatment did not result in any exacerbation. Her VA remains 20/32 in both eyes, and IOP is $17 \mathrm{~mm} \mathrm{Hg}$ in both eyes (under topical treatment). The macular edema has improved, but still remains significant and is likely the cause of her impaired VA (fig. 2).

\section{Discussion}

$\mathrm{TNF} \alpha$ has a significant role in the pathogenesis of granulomas; TNF $\alpha$ antagonists have been reported to be effective in the treatment of refractory sarcoidosis [6]. It is therefore paradoxical that such agents would also induce sarcoidosis, but this complication has been previously documented $[1,2,4]$. TNF $\alpha$-induced sarcoidosis may not demonstrate all the systemic manifestations of the disease and may involve only 1 organ. Several case reports 
Moisseiev et al.: Certolizumab-Induced Uveitis: A Case Report and Review of the

have been published describing pulmonary [7-9] or cutaneous [10,11] sarcoidosis following treatment with TNF $\alpha$ antagonists.

Certolizumab pegol (Cimzia) is a new TNF $\alpha$ antagonist. It is a recombinant, polyethylene glycolylated, antigen-binding fragment of a humanized monoclonal antibody that selectively targets and neutralizes TNF $\alpha$ [5]. Infliximab and adalimumab are monoclonal antibodies directed toward $\mathrm{TNF} \alpha$, and etanercept is a TNF-receptor-fusion protein conjugated to the Fc portion of human IgG. Certolizumab is essentially different from these other TNF $\alpha$ antagonists since it is not a full antibody and does not include an Fc portion that may be immunogenic.

Our patient had no previous history of sarcoidosis and had not received any other TNF $\alpha$ antagonists that may have induced it. The patient presented with 4 ocular signs of sarcoidosis (synechiae, vitreous opacities, multiple peripheral chorioretinal lesions and a bilaterality of involvement), associated with a mildly elevated ACE. According to the international criteria for the diagnosis of ocular sarcoidosis [12], 3 suggestive intraocular signs and 2 positive investigational tests are required to diagnose a probable ocular sarcoidosis. Since our patient had no other typical signs and only 1 positive test, a diagnosis of ocular sarcoidosis could not be made with any certainty, but it was evident that significant bilateral uveitis was present and the constellation of ocular findings and elevated ACE are suggestive of ocular sarcoidosis. The elevated IOP is typical and also supports this diagnosis.

The fact that the onset of ocular involvement followed the initiation of certolizumab supports the observation that the uveitis was induced by this agent and improved after its cessation. Although uveitis is a possible complication of RA, the ocular findings in this case are not typical for RA, but instead are highly suggestive of a diagnosis of sarcoidosis. It is worthwhile to note that the simultaneous occurrence of RA and sarcoidosis is extremely rare [1]. Also, the patient had never had any ocular involvement associated with RA. These facts further imply certolizumab as the cause of induced uveitis in this patient. Following cessation of certolizumab, the active inflammation subsided and the vitreous cleared in both eyes. The macular edema did not resolve completely; however, residual edema may persist after the inflammatory process is stopped, and in this patient, it is also possible that the presence of an epiretinal membrane precluded its complete resorption. The patient had a normal chest X-ray and pulmonary function; only her eyes were involved in the induced inflammation. For this reason, there was also no way to perform a biopsy to pathologically demonstrate the diagnosis of sarcoidosis. It should be noted that sarcoidosis may involve any organ [3] or it can be limited to the eyes only [12]. The same is true for induced sarcoidosis, which may be limited to 1 organ [4,7-11]. For example, it has been reported to present very atypically and involve just the hypopharynx [13].

This is the first case report of induced uveitis, suggestive of ocular sarcoidosis, secondary to treatment with certolizumab. Ocular involvement associated with autoimmune disease induced by TNF $\alpha$ antagonists is relatively rare [2], and ocular involvement from $\mathrm{TNF} \alpha$-induced sarcoidosis has only been reported once [4]. Cessation of certolizumab resulted in gradual improvement and should be recommended in similar cases; its use caused a new autoimmune disease. It should be noted that the patient required additional topical treatment as well as periorbital injections of steroids; extraocular involvement may have required systemic therapy. Rheumatologists and ophthalmologists should be aware of the possibility of induced uveitis by TNF $\alpha$ antagonists, and this case report demonstrates that this complication may also occur with certolizumab. Rheumatologists should also be aware that it may only involve 1 organ and inquire about changes in VA. They should refer their patients for an ophthalmic examination if any ocular involvement is suspected. 


\begin{tabular}{l|l}
\hline Case Rep Ophthalmol 2014;5:54-59 \\
\hline DOI: 10.1159/000358324 & $\begin{array}{l}\text { @ 2014 S. Karger AG, Basel } \\
\text { www.karger.com/cop }\end{array}$
\end{tabular}

Moisseiev et al.: Certolizumab-Induced Uveitis: A Case Report and Review of the Literature

\section{Disclosure Statement}

No author has any proprietary interest in the publication of this report. The authors declare no conflict of interest.

\section{References}

1 Tong D, Manolios N, Howe G, Spencer D: New onset sarcoid-like granulomatosis developing during anti-TNF therapy: an under-recognised complication. Intern Med J 2012;42:89-94.

-2 Ramos-Casals M, Brito-Zeron P, Soto MJ, Cuadrado MJ, Khamashta MA: Autoimmune diseases induced by TNF-targeted therapies. Best Pract Res Clin Rheumato 2008;22:847-861.

- Iannuzzi MC, Rybicki BA, Teirstein AS: Sarcoidosis. N Engl J Med 2007;357:2153-2165.

-4 Clementine RR, Lyman J, Zakem J, Mallepalli J, Lindsey S, Quinet R: Tumor necrosis factor-alpha antagonistinduced sarcoidosis. J Clin Rheumatol 2010;16:274-279.

5 Deeks ED: Certolizumab pegol: a review of its use in the management of rheumatoid arthritis. Drugs 2013;73:75-97.

6 Keystone EC: The utility of tumour necrosis factor blockade in orphan diseases. Ann Rheum Dis 2004;693(suppl 2):ii79-ii83.

7 Toussirot E, Pertuiset E, Kantelip B, Wendling D: Sarcoidosis occurring during anti-TNF- $\alpha$ treatment for inflammatory rheumatic diseases: report of two cases. Clin Exp Rheumatol 2008;26:471-475.

-8 Bhamra K, Stevens R: Pulmonary sarcoidosis following etanercept treatment. Case Rep Rheumatol 2012;2012:724013.

\9 Gifre L, Ruiz-Esquide V, Xaubet A, Gómez-Puerta JA, Hernández MV, Sanmartí R: Lung sarcoidosis induced by TNF antagonists in rheumatoid arthritis: a case presentation and a literature review. Arch Bronconeumol 2011;47:208-212.

10 Dhaille F, Viseux V, Caudron A, Dadban A, Tribout C, Boumier P, Clabaut A, Lok C: Cutaneous sarcoidosis occurring during anti-TNF-alpha treatment: report of two cases. Dermatology 2010;220:234-237.

11 Fok KC, Ng WW, Henderson CJ, Connor SJ: Cutaneous sarcoidosis in a patient with ulcerative colitis on infliximab. J Crohns Colitis 2012;6:708-712.

12 Herbort CP, Rao NA, Mochizuki M, members of Scientific Committee of First International Workshop on Ocular Sarcoidosis: International criteria for the diagnosis of ocular sarcoidosis: results of the first International Workshop on Ocular Sarcoidosis (IWOS). Ocul Immunol Inflamm 2009;17:160-169.

-13 Christoforidou A, Goudakos J, Bobos M, Lefkaditis E, Vital V, Markou K: Sarcoidosis-like granulomatosis of the hypopharynx as a complication of anti-TNF therapy. Am J Otolaryngol 2013;34:268-272. 
Case Reports in

Ophthalmology
Case Rep Ophthalmol 2014;5:54-59

DOI: 10.1159/000358324

Moisseiev et al.: Certolizumab-Induced Uveitis: A Case Report and Review of the Literature
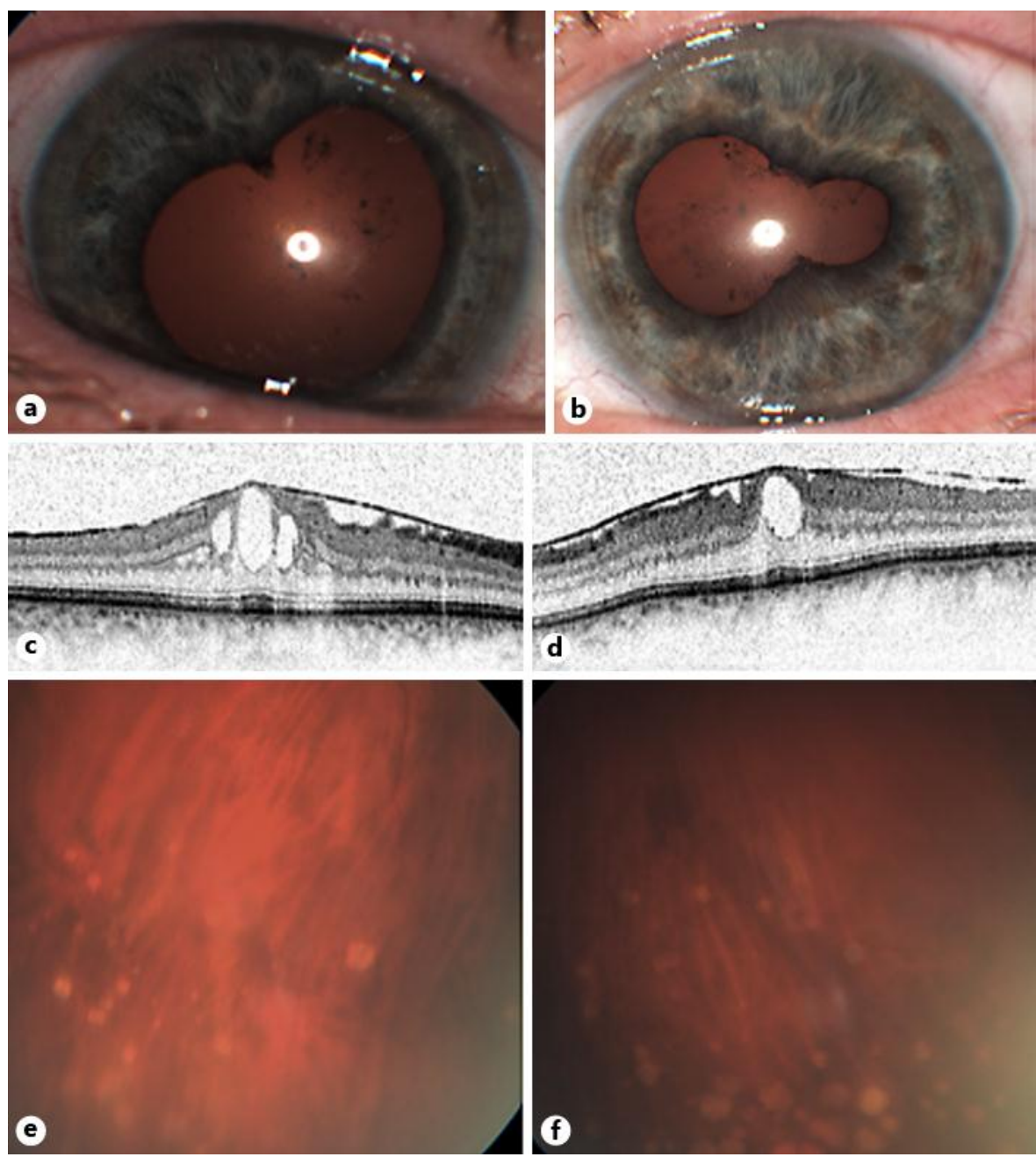

Fig. 1. Ocular findings at initial presentation included bilateral posterior synechiae (a, right eye; b, left eye), macular edema (demonstrated by OCT, c, right eye; d, left eye), and peripheral retinal punched-out lesions (e, right eye; $\mathbf{f}$, left eye). 
Moisseiev et al.: Certolizumab-Induced Uveitis: A Case Report and Review of the
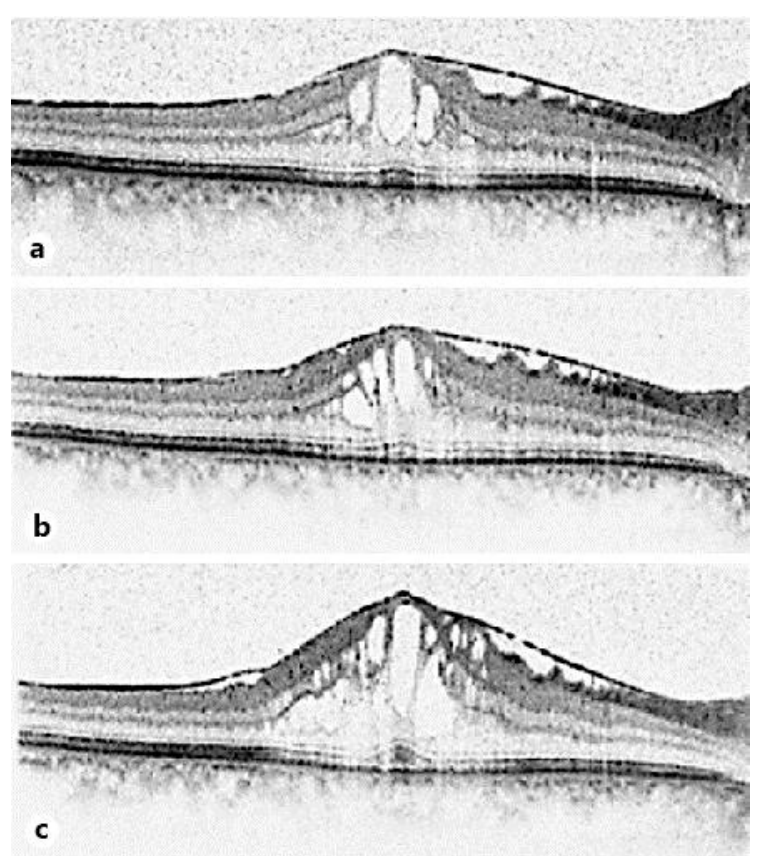

C

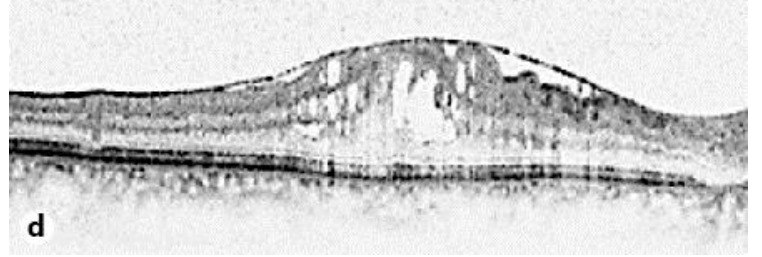

Fig. 2. Progression of the macular edema can be seen on OCT scans of the right eye (findings were similar in the left eye). At presentation (a), a significant macular edema was demonstrated, with an epiretinal membrane and mild vitreal opacification. Findings were stable at 3 months (b), but a worsening of the macular edema was noted at 6 months (c), and treated with an intravitreal injection of triamcinolone acetonide. Despite treatment and cessation of certolizumab, the macular edema persisted at 1 year (d). 